\title{
Les unions « interraciales » au Brésil : évolutions démographiques et interprétations sociologiques
}

version d'auteur pré-publication

\author{
Christophe Brochier ${ }^{1}$
}

L'étude de l'évolution dans le temps du nombre de couples appariant des individus de couleurs différentes ${ }^{2}$ est une thématique ancienne des sciences sociales au Brésil, puisqu'elle commence à la fin des années 1930 à São Paulo. L'analyse de la question est depuis régulièrement mise à jour par les démographes et les sociologues, même si elle concentre beaucoup moins d'attention que l'analyse des formes et des causes de l'inégale répartition des individus dans les strates de revenus selon leur couleur de peau (ou leur identité raciale supposée). Les constats que permettent ces études sont intéressants et importants pour comprendre l'évolution de la société brésilienne et l'on peut supputer que le relatif déficit d'attention à leur égard vient des difficultés d'interprétation. J'essaierai dans ce texte de dresser un état des lieux à la fois des faits que les démographes ont apportés et des analyses que les sociologues ont proposées. J'avancerai en fin de texte quelques suggestions sur la base notamment d'une enquête personnelle réalisée entre 2009 et 2013 et consistant à catégoriser l'écart phénotypique d'un échantillon important de couples cariocas. L'un des buts de cet article est de proposer l'idée que la façon dont vivent ensemble les Brésiliens selon leur couleur de peau ne peut être résumée à l'aide de formules tranchées et idéologiques, fréquemment utilisées depuis une vingtaine d'années dans les publications spécialisées, et

\footnotetext{
${ }^{1}$ Maître de conférences en sociologie, université Paris 8, chercheur au CREDA (IHEAL-Paris 3).

${ }^{2}$ La thématique « raciale » est peu familière au public français, mais représente une banalité pour les Brésiliens. En Europe et aux Etats-Unis, l'homme ou la femme de la rue a en général une vision biologisée des « races ». Les chercheurs, eux, adoptent plutôt le concept de « races sociales » : c'est-à-dire le résultat, dans les usages, de la croyance en l'existence de races biologiques. J'adopterai dans ce texte cette perspective et utiliserai le terme sans guillemets. De même je parlerai d'unions « interraciales » comme le font les Brésiliens pour désigner des couples dont les conjoints ne peuvent être rangés dans la même catégorie de couleur du recensement. Le lien entre race et couleur est mal conceptualisé au Brésil et se trouve soumis au plus grand flou et à la plus grande hétérogénéité, même chez les spécialistes. Il en va de même pour les races en tant que groupes sociaux, comme on le verra plus loin.
} 
déclinant 1'hypothèse d'un racisme généralisé gouvernant tous les aspects de la vie brésilienne ${ }^{3}$.

\section{La description du phénomène par les sociologues jusqu'aux années 1980}

Le Brésil publie depuis 1872 des recensements de population partiels ou complets qui ont régulièrement utilisés des items décrivant la race ou la couleur ${ }^{4}$. La question n'est pas posée en 1900, 1920 et en 1970 et les systèmes d'enregistrement ont considérablement varié au fil des ans en fonctions d'impératifs techniques, politiques, ou scientifiques. La comparaison des résultats d'une date à une autre est ainsi particulièrement hasardeuse ${ }^{5}$. Parmi les différentes formules utilisées, cependant, les catégories « noirs », « blancs » et « gris » (ou « métis ») ont, à quelques différences près, toujours été maintenues. La logique de ces enregistrements a cependant considérablement évolué. Au début du XXe siècle, il s'agissait de décrire racialement le Brésil en fonction des critères de l'anthropologie de l'époque. A partir de 1940, la question du sens de ces notions s'est posée pose et on a parlé de " couleurs » plus que de races. En 1970, comme on l'a dit, l'enregistrement de la couleur est abandonné. Il est repris à partir des années 1980 en réhabilitant la notion de race moins pour des raisons anthropologiques qu'en fonction des besoins d'une sociologie des inégalités.

Les sociologues n'ont cependant pas cessé de débattre de la signification des ces catégorisations dans la mesure où les chiffres enregistrent des auto déclarations dont le sens peut varier d'une classe sociale et d'un Etat à l'autre au sein d'une société très largement métissée mais fondée sur des histoires régionales très différentes. Il est ainsi évident que les Brésiliens métissés ne se voient pas comme «gris » et que la déclaration de couleur subit l'influence de l'histoire familiale, de la sociologie locale et des aléas de l'identité personnelle 6 . Il n'en demeure pas moins que depuis la fin des années 1970, les statistiques « raciales» sont de plus en plus utilisées par les sociologues et les anthropologues, notamment pour apporter des éléments de preuve d'un «racisme à la brésilienne » ${ }^{7}$. La question des unions ou des mariages racialement mixtes occupe une partie réduite de ces débats. Le faible nombre de travaux sur ce sujet, pourtant fondamental pour une « sociologie des relations raciales », s'explique sans doute par le fait que les chiffres sont difficiles à expliquer et qu'en outre ils montrent une augmentation des unions mixtes, ce qui coïncide mal avec la thèse désormais dominante du Brésil comme pays raciste.

Les sociologues brésiliens ou américains qui ont étudié la question jusqu'aux années 1960 au moins ne disposaient pas de données solides ou même utilisables, ce qui montre que les

\footnotetext{
${ }^{3}$ La plus claire formulation de ce type de perspective se trouve dans Thomas Skidmore Blacks into white, Duke University Press, 1974.

${ }^{4}$ Pour plus de précision: José Luis Petruccelli \& Ana Lucia Saboia (org), Caracteristicas étnico-raciais da população : classificações e identidades, Rio de Janeiro, IBGE, 2013.

${ }^{5}$ Ainsi en 1872, les esclaves furent enregistrés par leur maître et les réponses n'étaient pas obligatoires. La catégorie caboclo utilisée en 1890 pour enregistrer les métis de blancs et d'Indiens a disparu ensuite, etc. Par ailleurs les pressions politiques au tournant du siècle demandaient aux statisticiens d'aboutir au portrait d'un Brésil «blanc ». Cela n'empêche pourtant pas certains sociologues de décrire des évolutions depuis le XIXe siècle, comme si les données étaient sûres et comparables.

${ }^{6}$ Des constats similaires ont été faits aux Etats-Unis alors mêmes que les catégories raciales y semblent plus fixes et mieux assumées. Voir à ce sujet: Aliya Saperstein, «Double checking the race box : examining inconsistency between survey measures and observed and sel-reported race », Social Forces, vol. 85, n 1,2004 , pp. 57-74.

${ }^{7}$ Voir en particulier : Carlos Hansebalg, Discriminação e desigualdades raciais, Rio de Janeiro, Graal, 1979.
} 
autorités de l'époque s'intéressaient moins à ce phénomène qu'à celui du comptage des différents stocks raciaux. L'américain Donald Pierson, dans son étude pionnière sur les relations raciales à Salvador commencée en 1935, en fut réduit à fouiller dans les registres locaux de l'Etat civil et à interpréter les données à l'aide de l'employé municipal ${ }^{8}$. Il en tira le chiffre de 3,3\% de mariages interraciaux entre 1933 et 1934, mais sans compter les conjoints «blanchis » c'est-à-dire considérés comme blancs uniquement à partir du ton de peau et pas du phénotype parental. Dans une autre étude réalisée un peu plus tard dans une bourgade du Nordeste $^{9}$, Pierson a de nouveau compté les mariages mixtes sur la base des classifications opérées par l'employé de mairie : 60, $7 \%$ des 315 unions conclues entre 1931 et 1947 ont concerné un homme blanc et une femme blanche, 16,8\% un homme métis et une femme blanche, 9,5\% un homme blanc et une femme métis, 9,5\% un homme métis et une femme métis, les autres cas comptant pour moins de 2\%. L'endogamie était de $72 \%$ et aucun mariage n'a concerné des partenaires noirs et blancs, mais l'employé était connu d'après Pierson pour « blanchir» les conjoints en les enregistrant.

Samuel Lowrie qui étudie les mariages à São Paulo à la fin des années 1930 est de même contraint à des comptages dans les jardins d'enfants et ne produit pas de données combinant couleur et mariages ${ }^{10}$. Dans les années 1950, René Ribeiro qui examine les unions mixtes dans le Pernambuco n'est pas non plus capable de fournir des tableaux croisés ${ }^{11}$, pas plus que Luis Costa Pinto qui fait le même travail à Rio de Janeiro ${ }^{12}$. Thalès de Azevedo, de son côté, compte au début des années $1950^{13} 34 \%$ d'unions endogames à Salvador (d'un point de vue racial) à partir de l'examen de 222 mariages enregistrés par l'état civil. Au milieu des années 1950, l'Américain Austin Staley pour sa thèse de doctorat doit lui aussi produire des données personnelles : il rencontre et interviewe 222 couples racialement mixtes dans cinq grandes villes du Brésil. Ces couples mixtes sont composés pour les hommes de $60 \%$ de blancs, $8 \%$ de "bruns" (morenos), $22 \%$ de «mulâtres » et 10\% de noirs. Les femmes sont : $40 \%$ de blanches, $18 \%$ de métisses claires, $16 \%$ de métisses foncés et $26 \%$ de noires ${ }^{14}$. Nelson do Valle Silva ${ }^{15}$, à partir des chiffres de Staley pour São Paulo (1948-1957) a calculé que les mariages étaient endogamiques à $96,6 \%(98,7 \%$ chez les blancs mais $70,1 \%$ chez les " gris »). Les hommes noirs en revanche sont 7,4\% à épouser des femmes blanches. Emilio Willems ${ }^{16}$ quand il étudie à la même époque la bourgade de Cunha dans l'état de São Paulo arrive à la conclusion que seul $0,7 \%$ des mariages unissaient des noirs et des blancs et que 83 $\%$ étaient endogamiques.

Toutes ces enquêtes signalent quatre faits qui avaient déjà été relevés par les voyageurs occidentaux au XIXe siècle : les unions informelles interraciales sont plus courantes que les mariages interraciaux, dans les deux cas ce sont des conjoints de milieux sociaux et de phénotypes proches, dans les couples interraciaux, l'homme est en général plus foncé que la femme, et la proportion de mariages mixtes se fait en raison inverse de la classe sociale des époux (ils sont en fait très rares dans la haute société).

\footnotetext{
${ }^{8}$ Donald Pierson, Brancos e prëtos na Bahia, São Paulo, Companhia Editora Nacional, 1967.

${ }^{9}$ Donald Pierson, Cruz das almas, Rio de Janeiro, José Olympio, 1966.

${ }^{10}$ Samuel Lowrie, " Racial and national intermarriage in a brazilian city », American Journal of Sociology, vol. $44, \mathrm{n}^{\circ} 5,1939$, pp. 684-707.

${ }^{11}$ René Ribeiro, "O the amaziado relationships and other aspects of the family in Recife (Brazil) », American Sociological Review, vol. 10, n 1,1945 , pp. 44-51

${ }^{12}$ Luis A. Costa Pinto, O negro no Rio de Janeiro, Rio de Janeiro, Cia Editora nacional, 1953.

${ }^{13}$ Thales de Azevedo, As elites de cor numa cidade brasileira, [or. 1955], Salvador, Edufba, 1996.

${ }^{14}$ Austin Staley, « Racial democracy in brazilian marriage : toward a typology of negro-white intermarriage in five brazilian communities », American Catholic Sociological Review, vol. 21, n 2, 1960, pp. 146-164.

${ }^{15}$ Nelson do Valle Silva, «Distancia social e casamento inter-racial no Brasil », Estudos Afro-Asiáticos, $\mathrm{n}^{\circ} 14$, 1987, pp. 54-84.

${ }^{16}$ Emilio Willems, Cunha : tradição e transição em uma cultura rural do Brasil, São Paulo, Secretaria da agricultura, 1947.
} 


\section{Constats démographiques depuis les années 1980}

En 1970, l'IBGE a abandonné la variable couleur dans son recensement démographique. Les données produites par cet organisme n'autorisent donc véritablement la production de tables de mariages interraciaux qu'à partir de 1980. Les travaux produits sur cette base permettent de constater que les unions au Brésil suivent une logique de proximité des caractéristiques raciales ou phénotypiques. L'article déjà cité de Nelson do Valle Silva montre que les mariages sont endogamiques à $79 \%$. Mais l'endogamie est beaucoup plus forte chez les blancs $(84 \%)$ que chez les noirs $(52,1 \%)$, les métis se situant entre les deux extrêmes $(77 \%)$. L'étude de Berquó ${ }^{17}$ montre que pour 1980, 84, $7 \%$ des femmes blanches avaient épousé un blanc, 77, $1 \%$ des femmes métisses avaient épousé un métis et $67 \%$ des femmes noires un homme noir. Dans le même temps, cependant, les proportions de couples " mixtes ", c'est-àdire présentant des différences de types raciaux notables ne sont pas négligeables : l'auteur les évalue à $19 \%$. Ces études confirment également que dans les cas de mariages inter couleurs, la femme est dans la majorité des cas $(57,5 \%)$ plus claire que son conjoint.

Plus récemment, des études plus systématiques ont permis de comparer l'évolution dans le temps des unions interraciales (officielles ou non) pour l'ensemble du pays. L'article de Heaton et Mitchell ${ }^{18}$, permet ainsi de constater un très net affaiblissement de l'endogamie chez les blancs (qui passe de 82, 1\% en 1991 en prenant le mari comme point de référence à $64,2 \%$ en 2008), et chez les noirs (de 43,6\% à 33, 9\%), quoique plus modéré chez les métis (de $70 \%$ à 66\%). La proportion d'hommes blancs qui épousent des femmes métisses a presque doublé (passant de 16,5\% à 31,3\%) tandis que les couples hommes blancs/femme noir ont triplé en proportion (passant de 1,4\% à 4,5\%). L'affaiblissement de l'endogamie chez les noirs s'est fait essentiellement au profit des femmes métisses ( 6 points entre 1991 et 2008). Les hommes métis, pour leur part se sont plus largement tournés vers les femmes noires (les couples HM-FN passent de 2,4\% à 4,5\% du total des couples). L'évolution dans le temps est donc très claire : les couples sont de moins en moins marqués par la similitude de couleur (ou de « race »).

Des études plus fines permettent de constater les taux d'endogamie de couleur d'une région à l'autre. Ainsi Petruccelli ${ }^{19}$ fournit les chiffres suivant pour 1998 : Nord 67,9 \% ; Nordeste 73,1 $\%$; Sud-Est 80,1\% ; Sud 87,3\% ; Centre-Ouest 67,8 \%. Les régions les plus endogamiques sont les plus «blanches» mais elles sont aussi les plus riches. Dans le Centre-Ouest, où les blancs sont moins nombreux, leurs unions ne sont endogamiques qu'a 69,5\%. Dans toutes les régions, l'endogamie a nettement baissé entre 1987 et 1998 (entre 3 et 5 points).

L'étude de Petruccelli montre cependant que les taux de célibats ne sont pas les mêmes d'un "groupe de couleur» à l'autre, en particulier chez les femmes. Ainsi seules 48,3\% des femmes noires sont mariées contre $59,1 \%$ chez les blanches et 55,4 \% chez les « grises ». On ne retrouve pas ce phénomène chez les hommes, ce qui peut indiquer que ces derniers peuvent

\footnotetext{
${ }^{17}$ Elza Berquó, « The demography of inequality : considerations on black people in Brazil », Brazilian Journal of population studies, vol.1, pp. 165-178.

${ }^{18}$ Tim Heaton et Coller Mitchell, "Changing intergroup boudaries in brazilian mariages : 1991-2008 », Journal of comparative family studies, vol. $43, \mathrm{n}^{\circ} 4,2012$, pp. 461-482. Les données varient d'une étude à l'autre selon la taille des villes et l'âge des individus sélectionnés.

${ }^{19}$ José Luis Petruccelli, « Seletividade por cor e escolhas conjugais no Brasil dos 90 », Estudos Afro-Asiáticos, vol. $23, \mathrm{n}^{\circ} 1,20012$, pp. 29-52.
} 
compenser leur couleur par leur activité professionnelle. On constate cependant que les femmes noires sont aussi plus souvent que les autres divorcées et surtout veuves $(10,8 \%$ chez les noires mais 7,3\% chez les «grises »). On peut donc postuler l'effet d'un racisme s'abattant sur la femme noire, mais on peut aussi expliquer ces chiffres par des facteurs sociaux courants dans les milieux les plus populaires partout dans le monde : indices de santé plus mauvais, forte mortalité, moindre durée des unions, etc. Cette interprétation est renforcée par le fait que les taux de veuvages sont divisés par 6 quand on passe des femmes sans instruction à celles ayant au moins 8 années de scolarité. De même, les femmes sans instruction sont $15,9 \%$ à être célibataires, alors que ce taux passe à 39,5\% chez les femmes instruites. Ce ne peut donc être la couleur seule qui explique les taux de célibats.

L'importance des différences de scolarité est considérable même si la plupart des auteurs continuent à interpréter les taux de mariages comme un phénomène " racial ». Pour plus de détail, Silva et Ribeiro ${ }^{20}$ ont proposé de calculer l'endogamie éducationnelle à partir de 5 niveaux d'études : 0 à 3 ans de scolarisation, 4 à 7 ans, 8 ans, 9 à 11,12 ou plus. De cette manière, ils calculent une endogamie éducationnelle de $79,2 \%$ en $1960,59,4 \%$ en 1980 et 48, $1 \%$ en 2000 qui est donc toujours inférieure à l'endogamie de couleur (qu'ils évaluent à $88 \%$ en 1960, $80 \%$ en 1980 et $69 \%$ en 2000). Le biais de ce mode d'évaluation concerne évidemment les tranches d'années d'études choisies : outre qu'on ne voit pas bien à quelle logique institutionnelle ou sociale elles correspondent, elles conduisent à calculer l'endogamie stricte dans un tableau à 25 cases, alors que l'endogamie de couleur est évaluée sur un tableau à 9 cases ! Si l'on refait les calculs avec une matrice éducationnelle à 9 cases (études primaires, secondaires et supérieures) on arrive à une endogamie éducationnelle de $94,3 \%$ en 1960 et de $70,2 \%$ en 2000 , soit des niveaux toujours supérieurs à ceux de l'endogamie de couleur. On peut donc conclure que la dominance de la logique raciale sur la sélectivité scolaire est un artefact statistique.

Ce type de manipulation statistique est courant dans les travaux sur le sujet ${ }^{21}$ et a sans aucun doute pour objectif de suggérer au lecteur que c'est la race qui gouverne les rapports sociaux au Brésil. Or on voit bien le contraire en examinant les chiffres. Ainsi que le reconnaissent Silva et Ribeiro, les barrières que représentent les différences de scolarité sont considérables quand on se place aux extrémités. Ainsi les mariages entre des hommes ayant étudié 6 ou 7 ans et des femmes ayant étudié plus de 12 ans ne représentent que $0,4 \%$ des unions. Mais il n'est en fait pas nécessaire de se placer aux extrémités : les mariages où l'un des époux a au moins 12 ans d'études et l'autre moins de 9 ans ne représentent que 1,8\% des unions. Ces résultats n'ont rien de surprenant dans un pays très stratifié socialement où jusqu'aux années 1980 une grande partie de la population pauvre était encore peu ou pas scolarisée. Il est évident qu'une grande partie de l'endogamie raciale ou de couleur montrée par les recensements cache en fait simplement une sélection culturelle et sociale dans la mesure où les populations noires sont aussi les plus pauvres et les moins dotées en capitaux scolaires.

Dans le cadre d'une autre approche méthodologique, certains travaux fondés sur des questionnaires ont proposé des constats intéressants pour les années 1990. La recherche collective publiée sous le titre Racismo cordial $^{22}$ donne ainsi les résultats d'un questionnaire étoffé adressé à des échantillons de 2000 à 3000 personnes. L'un des principaux mérites des tableaux proposés est de tenir compte des relations amoureuses interraciales. Ainsi, $52 \%$ des

\footnotetext{
${ }^{20}$ Nelson do Valle Silva \& Carlos Costa Ribeiro, « Cor, educação e casamento no Brasil 1960 a 2000 », Dados, vol. 52, n¹, 2009, pp. 7-51.

${ }^{21}$ Dans d'autres travaux, les taux d'endogamie sont comparés avec un nombre de catégories différentes pour la couleur, l'âge et la scolarité. Ainsi, Fernanda Fortes de Lena (« Padrões de seletividade relacionados aos casais homo e heterosexuais no Brasil » Revista Brasileira de Estudos Populacionais, vol. 32, $\mathrm{n}^{\circ} 1,2015$, pp. 121137 ») utilise 3 catégories pour la couleur, 4 pour les niveaux d'études et 6 pour l'âge. Si on refait les calculs avec 3 catégories pour l'âge, l'endogamie provoquée par cette variable est proche de $80 \%$.

${ }^{22}$ Cleusa Turra \& Gustavo Venturini (org.), Racismo cordial, São Paulo, Ed. Atica, 1998.
} 
blancs, $58 \%$ des métis et $69 \%$ des noirs disent avoir eu au moins une relation amoureuse avec un partenaire d'une couleur différente. Ces chiffres sont considérables et suggèrent que l'endogamie raciale est surtout un effet du mariage. Les répondants ayant eu une relation amoureuse interraciale mais ne s'étant pas engagés dans une union mixte sont à peu près aussi nombreux d'un " groupe » à l'autre (40\% des répondants blancs, $40 \%$ des répondants métis et $43 \%$ des répondants noirs). La taille des « groupes » de couleur dans la population pauliste n'empêche donc pas les répondants de s'aimer en dépit des différences de couleur. En revanche seulement $12 \%$ des blancs, $18 \%$ des métis et $26 \%$ des noirs ont épousé un individu d'une autre couleur. C'est bien le mariage qui fait une différence et on peut tenir pour acquis que deux facteurs jouent à coup sûr : le niveau culturel et professionnel et la prévision de l'apparence physique des enfants. L'étude des relations amoureuses interraciales montre également que les femmes y sont, dans l'ensemble, moins ouvertes que les hommes, et les jeunes générations plus que les anciennes.

\section{Constats démographiques et méthodogenèse}

En matières d'analyses raciales, plus encore que dans n'importe quel type de description des populations, il convient de pouvoir évaluer, même sommairement, les effets possibles du recueil et du traitement des données, que j'appellerai ici méthodogenèse. Dans cette direction, il faut d'abord relever que les enquêtes sur la population utilisent au moins depuis 1980, comme on l'a vu, des catégories fixes proposées par l'IBGE. Si le blanc, le jaune et le noir ont du sens dans la vie courante pour parler de la couleur de peau, le terme " gris », rassemble en fait toutes les déclarations de couleur qui ne rentrent pas dans les catégories précédentes. On sait que les sondeurs ont souvent (surtout avant les années 1980) participé à l'élaboration des réponses en poussant les sondés à accepter les catégories officielles. Il n'en demeure pas moins que les frontières entre les catégories sont dans la réalité très poreuses. La différence entre «brun » et «blanc », dans la vie courante dépend des situations de déclaration (devant le sondeur ou chez l'esthéticienne, etc.). Il en va de même pour les nuances du " mulâtre » au «noir», qui de toute façon font entrer d'autres critères que la couleur (notamment les cheveux, le nez, etc.). Dans les lieux très métissés comme Rio de Janeiro ou Recife, la plupart des gens savent dire ce qu'ils ne sont pas mais (surtout les hommes) cherchent peu à élaborer une définition phénotypique fixe d'eux-mêmes. On constate fréquemment lorsque l'on pose des questions sur ce sujet que les répondants hésitent comme s'ils n'avaient jamais véritablement cherché à se définir par la race ou la couleur. Ils mettent en général en avant des descriptions physiques parfois complexes d'eux-mêmes mobilisant le riche vocabulaire brésilien au sujet de la peau, des cheveux, des traits, de la beauté, et faisant intervenir la description physique du reste de la famille. Les larges catégories du survey produisent ainsi une conceptualisation par en haut qui «réduisent une identité complexe en un tout uniforme $»^{23}$. Considérer que les déclarations lors des recensements donnent une photographie de la taille et de la localisation des " groupes de couleur » n'engage que les sociologues qui veulent bien le croire.

La perception des couleurs dépend aussi de la région et de la classe sociale. De nombreux couples catégorisés comme mixtes blancs/gris dans les Etats où l'immigration européenne a été massive comme à São Paulo ou dans le Paraná, seraient sans doute décrits comme

\footnotetext{
${ }^{23}$ Chinyere Osuji, «Confronting whitening in an era of black consciousness : racial ideology and Black-White interracial marriages in Rio de Janeiro », Ethnic and racial studies, vol. 36, n 10, 1490-1502.
} 
homogames dans une région comme Bahia où les noirs sont au contraire très nombreux. L'inverse est sans aucun doute également vrai : à Salvador, les différences entre tons de peau sombre ont plus d'importance qu'à São Paulo et un couple «mulato + negro », serait sans doute vu comme endogame à Porto Alegre. Les études sociologiques et démographiques n'intègrent pas les conséquences des écarts régionaux dans les analyses globales. Il en va de même pour l'effet des classes sociales. Tous les observateurs ont noté depuis le XIXe siècle que les membres des catégories moyennes et supérieures ont plus facilement tendance à « clarifier » leur ton de peau que les membres des classes populaires quand ils parlent d'euxmêmes. Cette simple tendance produit à coup sûr un grossissement artificiel de la proportion de blancs chez les strates qualifiées et donc, probablement une augmentation de l'endogamie raciale dans ces milieux, de même que dans les groupes sociaux les plus populaires par le phénomène inverse.

Dans le cas des unions interraciales, c'est en général le répondant qui donne la couleur de son conjoint lors des enquêtes. On peut assez logiquement supposer que les sondés auront tendance à réduire l'écart phénotypique au moment de répondre. Il n'existe pas, à ma connaissance d'étude qui essaie de vérifier, de mesurer ou même d'évaluer grossièrement les conséquences possibles de cette tendance. On peut cependant faire certaines suppositions plausibles concernant les données officielles de 2008. Ainsi il y aurait 64,2\% d'hommes blancs mariés à une femme blanche. On peut assez logiquement supposer qu'une partie de ces «blanches » peuvent être décrites dans d'autres situations comme des métisses, et que le chiffre est artificiellement gonflé en fonction de la situation d'enquête, aux dépends des métisses. A l'inverse, on peut supposer que le pourcentage des hommes blancs mariés à une femme noire est sous-estimé au profit des métis. Il est probable, de la même manière, que le chiffre des hommes noirs mariés à une femme noire est un peu gonflé au détriment des femmes métisses. Il n'est pas possible de calculer les écarts avec la "réalité » (si tant est qu'elle existe), mais cela ne signifie pas qu'il faille accepter les chiffres officiels tels qu'ils sont donnés.

Enfin, les déclarations de couleur sont sensibles au climat idéologique du moment. Jusqu'aux années 1980 (mais plus fortement encore avant guerre), la hiérarchie sociale et symbolique des types physiques et raciaux pénalisait fortement les signes de négritude. On peut, sans grand risque de se tromper, partir du principe que tous les recensements ont alors minimisé la proportion de noirs, en raison des effets de l'intériorisation des codes sociaux dans les déclarations. Depuis les années 1990, on assiste à un spectaculaire «black revival » au moins dans les grandes villes. La culture et l'identité afro-brésilienne sont d'autant plus valorisées que la lutte contre le racisme est devenue une priorité nationale. De ce fait, il est probable que l'augmentation de la proportion de noirs dans la population brésilienne lors des recensements est le produit d'un effet de déclarations revendicatives. On peut sans doute interpréter de cette manière une partie de l'affaiblissement de l'endogamie exposée précédemment. Par exemple la nette augmentation des couples HB/FN (qui passe de 2,4\% à 4,5\%). Mais il s'agit bien sûr de suppositions, toute certitude étant pratiquement inaccessible dans ce domaine.

La méthodogenèse prend aussi la forme du sens imposé aux mariages. Quand une différence de couleur est enregistrée entre les conjoints, le mariage est considéré comme «racialement mixte ", alors mêmes que souvent, les couples concernés ne voient pas les choses de cette manière ${ }^{24}$. Implicitement, le statisticien fait des conjoints les gestionnaires d'une situation de relations raciales, définissant de cette manière leur relation et laissant de côté les autres déterminants et critères donnant sens au couple. Cet inconvénient est d'ailleurs un trait permanent des études de sociologie des relations raciales qui ne voient les «noirs» que

\footnotetext{
${ }^{24}$ Osuji, art. cit.
} 
comme des « noirs » et les «blancs » que comme des «blancs » et traite comme " relation raciale » chaque forme de leur contact (voisinage, amitié, disputes, etc.) ${ }^{25}$.

Une alternative aux problèmes de fixité artificielle des groupes et de relativité des auto déclarations peut être trouvée dans des enquêtes qui mesureraient l'écart de couleur (et non pas les taux de croisement entre catégories fixes) entre les conjoints à partir d'une observation extérieure faite par un observateur unique. Il est bien sûr impossible de généraliser cette méthode à l'étude de la population brésilienne dans son ensemble, mais il est envisageable de réaliser des enquêtes par observation d'échantillons réduits dans certains centres urbains importants.

J'ai procédé à une telle enquête à Rio de Janeiro entre 2009 et 20013. En procédant par observation dans les rues de Rio de Janeiro, j'ai classé les couples croisés dans la rue ${ }^{26}$ selon le degré de proximité de leur couleur de peau : 1) identique ou quasi-identique, 2) petite différence (par exemple, phénotype métis clair + phénotype métis foncé), 3) grande différence (par exemple phénotype " métis foncé » + phénotype «blanc »). Le caractère subjectif d'une telle évaluation est compensé par le fait que la subjectivité est toujours la même et évite les enjeux égotiques de l'auto classification.

Les résultats finaux ont été les suivants : phénotypes identiques ou presque : 75,2 \%, phénotypes faiblement différents : 15,2\% ; phénotypes fortement différents : 9,6\%. Les taux d'identiques sont plus faibles et les taux de grande différence sont plus forts dans les quartiers populaires, où les noirs et métis foncés sont plus nombreux. Cette donnée est congruente avec les constats anciens faits par Pierson ou Azevedo indiquant que les mariages entre personnes présentant un écart de couleur important sont généralement plus fréquents dans les milieux populaires. En procédant de cette manière on évite de faire entrer la réalité dans les catégories de pensée rigides de l'exogamie et de l'endogamie. Rien ne prouve en effet que les Brésiliens raisonnent en termes de groupes de couleurs fixent ou même agissent sans le formuler explicitement en fonction de l'existence supposée de tels groupes. Les acquis généraux de la sociologie (internationale) du choix du conjoint sont que la fixation des couples se fait en recherchant la proximité sociale, culturelle mais aussi physique, ethnique, religieuse et raciale. Ce qu'il faut mesurer est donc l'écart phénotypique (entre autres choses) entre les conjoints et pas leur tendance à franchir de supposées «barrières ». Les chiffres de cette enquête sont en revanche congruents avec les taux généraux d'endogamie calculés pour le Brésil (autour de $75 \%)$.

\section{Les interprétations sociologiques « classiques » des constats démographiques}

Les premiers sociologues à s'être intéressés aux mariages interraciaux ont tâché de dégager un ensemble de logiques sociales pouvant expliquer à la fois l'endogamie globale et les taux non négligeables d'exogamie raciale. La forte proportion d'unions mixtes au Brésil en comparaison d'autres pays est bien sûr le résultat d'un mode de peuplement particulier depuis

\footnotetext{
${ }^{25}$ On pourra trouver des exemples de cette tendance y compris dans les études qualitatives d'auteurs comme Florestan Fernandes, Antonio Guimarães ou Jeferson Bacelar.

${ }^{26}$ Les Brésiliens en couple se donnent en général la main ou manifestent des attitudes d'intimité. Je n'ai intégré à mon échantillon que les hommes et les femmes qui avaient de tels gestes ou ceux accompagnés d'enfants les appelant «pai » ou « mãe ». Au total mon échantillon était composé de 2023 couples, observés dans toutes les zones de la ville.
} 
le XVIe siècle: peu de femmes blanches et beaucoup de femmes indiennes et noires facilement accessibles sexuellement aux hommes d'origines portugaises. Ces conditions ont parfaitement été expliquées par les historiens et les sociologues comme Gilberto Freyre et Caio Prado Jr. Le système législatif et les mœurs ne se sont jamais vraiment opposées aux unions interraciales (surtout non formelles), même si ces dernières n'étaient pas regardées positivement ${ }^{27}$ et les mariages interraciaux analysés aujourd'hui se font donc dans un pays où la population métissée est déjà très abondante.

A partir de ce constat, Donald Pierson qui a été le premier chercheur à proposer une véritable étude de "relations raciales $»^{28}$ au Brésil, a cherché à comprendre pourquoi les unions mixtes n'étaient pas plus nombreuses. Son idée était que les Brésiliens ne manifestent pas un dégoût racial pour les individus au phénotype africain (comme c'était le cas aux Etats-Unis), mais que dans la société brésilienne, le " noir » est surtout une cristallisation sociale de l'individu de basse extraction. Alors qu'aux Etats-Unis, les classes populaires sont particulièrement hostiles (à son époque tout au moins) aux unions mixtes, au Brésil c'est chez les classes supérieures que les réticences sont les plus fortes. Pour lui, même s'il existe à l'évidence dans ce pays, qui se veut une extension de l'Europe, une hiérarchie symbolique des types physiques, c'est surtout la coïncidence des lignes de couleur et de classes (les noirs sont pauvres et les riches sont blancs) qui explique la relative faiblesse des unions mixtes. Les mariages mixtes sont selon lui d'autant plus fréquents qu'ils concernent des gens au phénotype proche et que d'autres éléments de statut social peuvent venir compenser l'écart de couleur. «En fait les mariages traversent les lignes de couleur plus souvent que les lignes de classes $»^{29}$. A l'appui de cette idée, il a examiné les 7 cas de mariages mixtes dans la bourgade de «Cruz das Almas » étudiée par son équipe dans les années 1950. Les conjoints étaient tous de classe populaire et ne semblaient pas avoir de concepts équivalents à la notion de «mariage interracial». Cette indifférence relative des paysans pauvres à la couleur du conjoint rejoint le tableau dressé par un autre observateur américain, l'anthropologue Ruth Landes dans les années $1940^{30}$. Selon ses observations, un homme de couleur appartenant aux classes supérieures se pense séparé par un abîme des «noirs » pauvres et ne peut se marier qu'à une femme de même milieu social, indépendamment de sa couleur. La désignation de « noir » s'adresse en fait aux gens pauvres de la rue et de la campagne et ne peut concerner les individus de mœurs aristocratiques. La barrière infranchissable est donc celle des classes, surtout dans les parties les plus hautes de la société ${ }^{31}$.

Le médecin et sociologue bahianais Thales de Azevedo est cependant celui qui a donné dans les années 1950 et 1960 l'analyse la plus poussée de la dynamique des unions interraciales ${ }^{32}$. Il rejoint les auteurs précités en insistant sur le fait que les catégories « noirs » et « blancs » ne désignent pas des groupes raciaux mais des groupes sociaux à la composition phénotypique

\footnotetext{
${ }^{27}$ Arthur Ramos, « Contacts of race in Brazil », Social Forces, 19, 4, 1941, 533-538.

${ }^{28}$ Les analystes peu familiarisés avec l'histoire de la sociologie confondent généralement les études qui abordent les questions de couleur et de race d'une manière ou d'une autre avec les études de « relations raciales ». Les réflexions sur les races au Brésil sont innombrables depuis le XIXe siècle au Brésil : elles cherchaient en général à évaluer les conséquences civilisationnelles de la coexistence de plusieurs races pour le « peuple brésilien ». Le concept de «sociologie des relations raciales» a été apporté au Brésil par les chercheurs américains, essentiellement Pierson, dans les années 1940 (Freyre n'est donc pas en 1933 un sociologue des relations raciales comme on le dit à tort très souvent). Il renvoie à l'étude des relations entre « groupes raciaux » en tant que tels. Pour les Etats-Unis l'existence de groupes raciaux constitués ne faisait pas de doute, mais la question se posait au Brésil. Pour Pierson, il n'y avait pas de vrais groupes raciaux au Brésil et donc pas, en fait, de " relations raciales ».

${ }^{29}$ Donald Pierson, «Brazilians of mixed racial descent », in : Noel Gist \& Anthony Dworkin, The blending of races, New York, Wiley, 1972.

${ }^{30}$ Ruth Landes, A cidade das mulheres, Rio de Janeiro, Editora UFRJ, 2002.

${ }^{31}$ On trouvera des analyses allant dans le même sens chez Felte Bezerra : Etnias Sergipanas, Aracaju, 1950.

${ }^{32}$ As elites de côr...op. cit.
} 
variable : «Blancs et noirs, dans ce contexte, sont des synthèses de rôles et de statuts joints à l'aspect physique ou phénotypique $»^{33}$. Il est aussi d'accord sur le fait que le parallélisme des échelles chromatiques et sociales ${ }^{34}$ incite en général les Brésiliens à chercher un conjoint au moins aussi clair qu'eux. De façon consciente ou inconsciente, ils cherchent par ce moyen à gagner en prestige social et pas à « purifier la race ». Trouver un conjoint plus clair est aussi un moyen de favoriser l'ascension sociale des enfants. Les Brésiliens et Brésiliennes que l'on interroge à ce sujet, selon Thales, présentent rarement les choses de cette manière et rationalisent prétendant que « ça c'est fait tout seul » quand ils trouvent un conjoint plus clair, ou bien à l'inverse, dissimulent leurs échecs sous l'angle du peu d'intérêt pour le mariage. Les hommes à la peau foncés trouvent plus facilement des conjoints clairs que les femmes à la peau foncée car ils peuvent compenser leur désavantage phénotypique par leur position sociale, leurs études, leur argent ou leur métier. A l'époque où Thales écrit, cela n'est guère possible pour les femmes, qui occupent des positions de relégations sur le marché du travail. Les hommes noirs en position de réussite choisissent donc souvent une femme blanche, même venant d'une famille moins riche ou moins respectée que la leur. La plus grande fréquence relative des couples HN/FB s'explique aussi par le fait que dans les traditions brésiliennes, c'est la famille de la femme qui « gagne un fils » lors du mariage. L'homme noir se blanchit donc par l'intermédiaire de sa belle famille. Plus la différence de couleur est grande, plus le conjoint foncé devra compenser par d'autres éléments du statut social. La couleur est l'un des éléments au poids négatif dans un complexe système de combinaisons attribuant aux individus un statut social dans le marché matrimonial. Dans les hautes sphères de la société et notamment les familles anciennes de propriétaires terriens, l'instinct de caste est si fort que seuls quelques métis très clairs issus de familles prestigieuses sont acceptés comme conjoints. Le modèle de Thales de Azevedo est assez finement complété par celui de l'anthropologue René Ribeiro, lui aussi élaboré pour le Nordeste ${ }^{35}$. Ribeiro part également du principe que la miscégénation est limitée par des barrières sociales et des patterns culturels hérités de l'époque esclavagiste. Ces barrières, fondées sur des préjugés de couleur agissent dans tous les milieux mais plus fortement dans les classes moyennes et supérieures. Il en résulte que même les hommes noirs pauvres essaient de se marier avec des femmes plus claires. Seuls parmi eux, les hommes âgés, très pauvres ou avec des enfants à charge y renoncent. Les autres recourent à la magie traditionnelle s'il le faut et promettent à leur fiancée plus claire une relation romantique. De ce fait, les femmes blanches, mêmes pauvres sont en position d'exiger un mariage officiel ou un décalage de richesse en leur faveur. Les femmes noires, pour leur part, ont plus de mal que les autres à trouver un conjoint, en dépit du mythe de leur attractivité sexuelle. Cette difficulté est encore plus grande pour celles venant de familles cultivées, et qui attendent de ce fait un conjoint de même niveau social. Au sujet des unions mixtes effectives, Ribeiro, sur la base de déclarations de personnes qui n'étaient pas directement impliquées, a insisté sur les difficultés que rencontrent les conjoints pour s'entendre entre eux et se faire accepter par leur entourage.

Le sociologue américain Austin Staley dans les années 1950, n'a pas proposé de modèle général mais a étudié dans le détail des cas de mariages mixtes dans plusieurs Etats de la fédération en interrogeant les conjoints eux-mêmes. On tire de ses comparaisons le constat, congruent avec les données de Pierson et d'Azevedo, que la plupart des mariages mixtes se font au sein du même groupe social. Mais surtout Staley montre, à la différence de Ribeiro, que beaucoup de mariages mixtes (presque la moitié) ne donnent lieu à aucun conflit familial. L'une des raisons, en plus de la proximité de classe ou de couleur de peau, est l'isolement

\footnotetext{
${ }^{33}$ Thales de Azevedo, «Mestiçagem e status no Brasil », Ve, colloquio internacional de estudos brasileiros, Lisboa, 1964, p. 12.

${ }^{34}$ J'emprunte l'expression à Caio Prado Jr, Formação do Brasil contemporraneo-colonial, São Paulo, Ed. Brasiliense, 1965, p. 105.

${ }^{35}$ René Ribeiro, « Situação étnica no Nordeste », Sociologia, vol. XV, n³, 1953, pp. 210-259.
} 
familial du conjoint blanc. Dans à peine $16 \%$ des cas, la différence de couleur est un problème non résolu pour le couple et les familles.

Ce sont en fait les études concernant São Paulo qui ont le plus insisté sur la faiblesse quantitatives des unions interraciales sur la base de l'idée d'un racisme systématique. Samuel Lowrie, en se fiant uniquement aux constats statistiques obtenus par ses enquêteurs, attribue le faible nombre d'unions mixtes à São Paulo au "préjugé racial ». Mais il n'apporte aucune preuve à cette affirmation comme le lui ont fait remarquer plusieurs critiques $^{36}$. La démonstration de Roger Bastide en 1953 suit à peu près les mêmes lignes. Comme Ribeiro et Azevedo, il se fonde sur des déclarations de « répondants » non identifiés et non caractérisés. Mais il le fait de façon à montrer que les mariages interraciaux sont rares en raison du racisme. Le mode opératoire de ce racisme n'est pas précisé hormis le fait qu'il limite les unions à des couples de couleurs proches. "Alors que dans le cas d'unions éphémères, c'est le blanc qui courre derrière la noire, pour le mariage, le cas le plus fréquent est celui d'un noir avec une blanche $\aleph^{37}$. Bastide insiste sur l'opposition des familles à ce type d'unions et cite les déclarations des interviewés selon lesquelles les femmes blanches qui épousent des noirs sont des « créatures qui ne valent rien ». « Toute la société blanche est solidaire contre la trahison à la couleur $»^{38}$. D'après ces exemples, les mariages mixtes sont en général malheureux. On comprend mieux le caractère sommaire et lapidaire de ces affirmations quand on sait que le livre de Bastide et Fernandes a été pensé comme un instrument de dénonciation du racisme à São Paulo et que l'enquête a été confiée à des militants des associations de défense des noirs. Une partie des données vient de sondages demandant aux répondants leur réaction à une proposition imaginaire de mariage interracial, ce qui n'est pas du tout la même chose que l'analyse d'unions effectives.

Si l'on excepte ce cas extrême, les analyses classiques (jusqu'aux années 1960) les plus solides tendent à montrer que les mariages interraciaux ne sont pas rares dans les régions à fort métissages dans les groupes populaires. Ils impliquent en général des partenaires proches socialement et physiquement. La dimension raciale est cependant toujours présente dans les calculs matrimoniaux puisque un phénotype africain dénote une infériorité sociale en raison du poids symbolique de l'esclavage. Le fait d'être noir, comme celui d'être illettré, pauvre ou d'origines inconnues est toujours un désavantage sur le "marché matrimonial». Ce désavantage peut cependant être compensé par un facteur positif (statut professionnel, réseau social, etc.) sauf dans les secteurs les plus traditionnels des classes dominantes qui ont clairement une mentalité de caste. Tous les analystes, y compris Bastide, sont d'accord pour reconnaître que les préjugés en jeu sont de l'ordre du mépris social et pas du dégoût biologique.

On peut toutefois constater que ce schéma, malgré son caractère convaincant, n'est pas sociologiquement très abouti. Ainsi les remarques, largement connues au Brésil, de Robert Merton sur le sujet ${ }^{39}$ n'ont pas donné lieu à un complément d'élaboration. Merton signale en effet que les sociologues ont tendance à confondre mésalliance et exogamie. La mésalliance est la rupture des règles (explicites ou non) gouvernant les « bons mariages ». L'exogamie désigne le choix d'un conjoint hors du groupe. Pour Merton, aux Etats-Unis, il existe des groupes raciaux, et donc une forme claire d'exogamie raciale. Une partie des analyses anthropologiques sur les unions raciales au Brésil traite les mariages mélangeant les couleurs comme des cas d'exogamie, considérant ainsi de facto qu'il existe des " groupes de couleur » au Brésil. Ces groupes correspondraient à ceux des statistiques et seraient assimilables à des

\footnotetext{
${ }^{36}$ Les remarques critiques de Rüdiger Bilden et de E.B. Reuter ont été publiées à la suite de l'article par l'American Journal of Sociology.

${ }^{37}$ Roger Bastide \& Florestan Fernandes, Brancos e negros em São Paulo, 3eme édition, p. 184.

${ }^{38}$ Idem, p. 186.

${ }^{39}$ Dans sa version brésilienne : Robert Merton, « Casamento e estrutura social », in : A ambivalencia sociologica e outros ensaios, Rio de Janeiro, Zahar, 1979.
} 
groupes sociaux c'est-à-dire qu'ils seraient gouvernés par des normes et appliqueraient des règles de fermeture. C'est pour le moins une hypothèse forte... Pierson qui est pratiquement le seul à avoir affronté complètement le problème avait clairement répondu par la négative. Or Merton montre bien que pour pouvoir parler de mariages inter groupal il faut non seulement que ces groupes existent en tant que tels dans la société, mais encore que les individus étudiés soient clairement affiliés à ces groupes et ne se limitent pas à posséder certaines caractéristiques des membres des groupes. Ainsi un mariage entre une fille d'Italiens et un fils d'Espagnols ne représente pas, faute de preuve, un mariage inter groupal. Aucune de ces subtilités n'est présente dans la plupart des analyses sociologiques sur le phénomène qui nous intéresse. Ainsi la mésalliance inter groupale ne devrait pas être confondue avec la mésalliance intra groupale. En outre, il faudrait déterminer quels sont les groupes et quelles sont les règles. Une hypothèse probable, mais jamais à ma connaissance discutée, ferait en fait des classes sociales les groupes et de la ressemblance physique la règle. Un mariage exogamique serait ainsi un mariage hors classe et une mésalliance, un mariage avec plus foncé que soi.

\section{Interprétations récentes}

Depuis les années 1980 ce sont surtout les sociologues quantitativistes et les démographes qui étudient les mariages mixtes. Ce phénomène se traduit par une tendance à inférer de façon plus ou moins assumée les relations réelles entre les individus à partir des constats statistiques sans pouvoir proposer un modèle anthropologique. Nelson do Valle Silva (article cité) est l'un des premiers auteurs de cette nouvelle vague. Il se propose en 1987 de tester la solidité de ce que j'ai appelé le modèle classique en examinant les chiffres du recensement de 1980 (alors même que le modèle classique avait lui-même été élaboré pour expliquer des chiffres). Il confirme les règles d'endogamie de couleur et de légère surreprésentation des couples où la femme est plus claire que son conjoint. Mais il ajoute également qu'en général le mari est plus scolarisé que la femme et qu'il ne semble donc pas exister de mécanisme de compensation de la couleur par le niveau d'éducation. Silva pose comme a priori, sur la base de travaux américains, que des « barrières » bloquent les mariages entre " groupes raciaux » différents et que ces barrières s'additionnent pour des unions entre groupes éloignés quels que soient le sexe et la couleur concernés. Il ne justifie pas ces affirmations par une observation « de terrain » des relations réelles. Il ne cherche pas non plus à élaborer un modèle anthropologique inductif à partir de cas observés par lui ou par d'autres. Il en va de même, depuis lors, pour la plupart des sociologues quantitativistes qui ont suivi son modèle. Leur préoccupation théorique est essentiellement de montrer que la «démocratie raciale ${ }^{40}$ n'existe pas au Brésil et que c'est en fait le « racisme » qui régit le rapport entre « groupes raciaux $\gg$.

Dans cette direction, l'une des explications possibles des phénomènes d'exogamie ou d'endogamie est la taille variable des groupes de couleurs. Tous les recensements montrent en

\footnotetext{
${ }^{40}$ Ce terme popularisé par Florestan Fernandes est en fait particulièrement flou et a contribué à ralentir les avancées en matière de sociologie des relations raciales au Brésil. Il désigne parfois une société sans caste (dans l'idée de Freyre), parfois une société sans racisme (le terme racisme étant en général laissé lui aussi dans le vague), parfois une société où la couleur n'a pas de rôle dans les relations sociales. Pour une mise en perspective et une critique : C. Brochier, «Le concept de 'démocratie raciale' dans l'histoire intellectuelle brésilienne », Revue de synthèse, vol. 135, $\mathrm{n}^{\circ} 1,2014$, pp. 123-150.
} 
effet que les blancs sont plus nombreux que les métis, eux-mêmes plus nombreux que les noirs, ce qui conditionne les possibilités d'appariement. Nelson de Valle Silva a donc calculé que si les groupes avaient la même taille (en 1980) les résultats finaux seraient légèrement différents. L'endogamie générale augmenterait (passant de $79 \%$ à $82 \%$ ) mais celle du groupe blanc baisserait de $84 \%$ à $77,9 \%$ alors que celle des noirs passerait de $52 \%$ à $83 \%$ (celle des métis passant de $77 \%$ à $71 \%$ ). Il en conclut que la tendance de fond est bien celle de la recherche du semblable. Ce type de raisonnement appelle bien sûr des critiques sociologiques assez évidentes. Il suppose que toutes choses restent égales par ailleurs au moment où l'on procède à la manipulation statistique (les usages, les représentations, la structure de classe, etc. ne seraient pas modifiés). Ensuite, les fréquences des mariages mixtes changeraient en proportion du changement de taille des groupes. Or, dans une société de caste, la taille des groupes n'a pratiquement pas d'incidence sur les quantités de mariages mixtes. Supposer une élasticité des mariages mixtes à la taille des groupes implique donc que l'on considère que les unions ne suivent pas des logiques de castes et qu'elles sont libres. On mesure donc les préférences raciales à partir d'hypothèses implicites sur la force de ces mêmes préférences. La règle de fonctionnement de ces mariages serait la recherche de la similarité physique, tempérée seulement par la disponibilité du partenaire adéquat. Ce modèle repose également implicitement sur l'idée que les mariages se font en fonction des stocks relatifs de population à l'échelle du pays. Dans les faits pourtant, les unions se font en fonction des lieux et des situations sociales des rencontres, déterminées localement. Enfin, ce raisonnement part du principe que les déclarations de couleur dans les recensements sont assez solides pour pouvoir parler de groupes de couleur (et donc de taille de ces groupes) au niveau national. Concrètement, cela signifie qu'un «blanc» déclaré dans le Maranhão appartient à un "groupe » différent qu'un métis déclaré dans la Paraná, etc. Cette supposition est bien évidemment douteuse, compte tenu de la plasticité locale des identifications de couleur en fonction des usages et des compositions démographiques de chaque région.

A partir des mêmes présupposés, Edward Telles arrive à la conclusion que puisque les mariages entre blancs et noirs resteraient minoritaires dans une société brésilienne théorique à tailles des "groupes raciaux » égales (contrairement au mariages incluant des métis), la mixité au Brésil cache en fait que le "racisme et l'inégalité sont grands ${ }^{41}$ ». Ce type de conclusion mélange un truisme et une affirmation floue et mal fondée et ne peut que laisser le lecteur dubitatif. Pourtant, quelques années plus tard, Petruccelli ${ }^{42}$ en se servant également de tableaux réajustés et à partir du constat (déjà ancien pourtant) que les femmes noires sont plus souvent célibataires que les femmes métis ou blanches, conclut que : « (...) en dépit de l'augmentation apparente [sic] de couples mixtes dans la population brésilienne, le maintien des frontières interraciales et la reproduction des inégalités sont assurés à partir de l'importance de la sélectivité maritale dans le comportement nuptial par groupes de couleur de la population brésilienne ». Encore une fois, ici, la recherche de la dynamique profonde des unions mixtes est abandonnée au profit de dénonciations confuses du «maintien de l'inégalité ».

La faiblesse de ces suppositions sociologiques ajoutées à la fin d'études descriptives démographiques vient essentiellement du fait que tout véritable schéma explicatif doit prendre position dans les débats particulièrement complexes portant sur les effets des différences de couleur et/ou de race dans la vie sociale brésilienne. Aucun démographe ne peut sérieusement supposer en quelques lignes de quelle manière la structuration de la société et la combinaison des caractéristiques sociales et raciales agit au niveau des relations entre individus et groupes

\footnotetext{
${ }^{41}$ « Racial distance and region in Brazil : intermarriage in urban areas », Latin American Research Review, vol. 28, n², 1993, pp. 142-162

${ }^{42}$ José Luis Petruccelli, « Seletividade por cor e escolhas conjugais no Brasil dos 90 », Estudos Afro-Asiáticos, vol. $23, \mathrm{n}^{\circ} 1,20012$, pp. 29-52.
} 
au niveau local et national. Cela est d'autant plus vrai que de nombreuses études quantitativistes projettent sur la réalité sociale les catégories et les raisonnements de la démographie. Ainsi, comme on l'a vu, les «groupes raciaux» sont jusqu'à preuve du contraire une invention commode des chercheurs dressant des tableaux statistiques, de même que les « barrières entre groupes », ou la « compensation raciale » supposée être mesurable. Il est évident en fait que les sociologues qui ne font pas d'enquête de terrain ignorent comment, dans les faits, les couples se forment. Ils tombent ainsi dans le travers régulièrement dénoncé notamment par Howard Becker ${ }^{43}$ qui consiste à mesurer l'effet de " variables » sélectionnées a priori au lieu de découvrir dans les faits toutes les forces à l'oeuvre ainsi que leur modes opératoires. A cela s'ajoute une intention militante tournée vers la recherche de la dénonciation du « racisme ». Or il n'est guère de concept plus flou dans les sciences sociales, en particulier au Brésil, où depuis les années 1930 les chercheurs essaient de caractériser le type de mépris qui correspondrait exactement aux combinaisons de caractéristiques sociales et phénotypiques (dans le cas où l'on pourrait séparer les deux).

Prenant le contre-pied de l'inférence des relations réelles à partir des constats statistiques, certains anthropologues de terrain ont suggéré que la compréhension des mariages mixtes devait se faire non seulement en tenant compte des normes sociales mais aussi des subjectivités des acteurs. Laura Moutinho ${ }^{44}$ par exemple a proposé de remettre en question l'explication structurelle des unions mixtes vues comme des outils tactiques de mobilité sociale $^{45}$. Selon elle, les statistiques "suggèrent des chemins, répètent ou écartent certaines hypothèses, mais ne mettent pas à jour les représentations et les valeurs sociales qui orientent ces choix $\rangle^{46}$. Zelinda Barros ${ }^{47}$ qui a conduit une enquête de même type auprès de couples mixtes bahianais refuse de même la solidification des identités raciales qu'opèrent les analyses statistiques. Les auto identifications sont beaucoup plus fluides, de même que les visions au sujet des mariages et de l'interracialité. Sur le terrain, les calculs mécanistes de compensation racialisée dans un «marché matrimonial» perdent de leur pouvoir explicatif. Ces études comme celles de Jordana Freitas de Sousa sur les unions mixtes à Fortaleza ${ }^{48}$ suggèrent également que les "problèmes causés par l'opposition des familles » peuvent être surévalués ou mal compris : ils sont parfois décrits comme inexistants comme l'avait vu Staley mais ils peuvent aussi matérialiser un préjugé des noirs envers les blancs. D'une manière générale, elles suggèrent que les configurations qui président à la réalisation et à la conduite des unions mixtes sont plus complexes qu'il n'y parait en examinant les recensements : jeu des carrières amoureuses, association de ruptures diverses au niveau des biographies, représentations sur les affinités esthétiques, rôle complexe des statuts sociaux de chaque famille, coupures d'une génération à l'autre, etc. Il est très probable que les modèles simplificateurs en termes de « barrières raciales », « racisme » et « calculs coûts/bénéfices sur un marché matrimonial » déforment fortement les dynamiques réelles.

\footnotetext{
${ }^{43}$ Howard Becker, La Bonne focale : de l'utilité des cas particuliers en sciences sociales, La découverte 2016.

${ }^{44}$ Laura Moutinho, Razão, cor e desejo, São Paulo, UNESP, 2004

${ }^{45}$ Elle s'oppose ainsi à des auteurs qui soutiennent cette théorie à partir d'une base empirique réduite, comme : Neusa Souza, (Tornar-se negro : as vicissitudes do negro brasileiro em asseçao social, Rio de Janeiro, Graal, 1983) ou Diva Moreira \& Adalberto Sobrinho («Casamento inter-raciais : O homem negro e a rejeição da mulher negra », in: Albertina Costa \& Tina Amado (org.), Alternativas escassas, saúde, sexualidade e reprodução na América latina, São Paulo, Editora 34,1994).

${ }^{46}$ Moutinho, op. cit., p. 27.

${ }^{47}$ Zelinda Dos Santos Barros, Casais inter-raciais e suas representaçoes acerca de raça, Thèse de doctorat en sociologie, UFBA, 2003.

${ }^{48}$ Jordana Freitas de Sousa, Afetos e relações inter-raciais na cidade de Fortaleza, Mémoire de fin d'étude en service social, Universidade Estadual do Ceará, date 2008.
} 


\section{Propositions d'analyses et de recherche}

Les critiques précédentes ne cherchent pas à montrer que la recherche sur les mariages mixtes est jusqu'à maintenant une entreprise vaine. Au contraire, je souhaiterais soutenir l'idée que ce domaine d'étude est fondamental pour la compréhension des relations raciales et sociales au Brésil. Il est cependant évident que l'orientation quantitativiste opérée à la fin des années 1970 dans la lignée de Carlos Hasenbalg est loin d'avoir résolu tous les mystères qui peuvent nous intéresser. Les sociologues des années 1950 disposaient de peu de chiffres mais cherchaient à les comprendre par des études de terrain. Nous avons aujourd'hui des constats statistiques abondants qu'il faut pouvoir relier à des observations anthropologiques.

Ainsi, il est possible de partir du «schéma classique» pour proposer des lignes de questionnement. En premier lieu, le rôle du milieu social comme facilitateur ou inhibiteur des mariages mixtes n'est qu'imparfaitement compris par les sociologues des années 1950 quand on s'éloigne des classes favorisées. Ces analystes connaissaient mal les classes populaires. La façon dont le statut social se combine à l'apparence physique et se voit interprété varie certainement du groupe des métayers à celui des petits paysans, des ouvriers immigrés à celui des ouvriers qualifiés locaux, etc.

Ensuite, il est évident que le fait d'être noir ou d'avoir des traits « africains » constitue une caractéristique négative pour la recherche d'un conjoint au Brésil. Le programme consistant, dans la lignée de Bastide et de Fernandes, à n'étudier les unions mixtes qu'afin de le dénoncer sous le nom de « racisme » doit être abandonné. Il est plus que temps aujourd'hui de chercher à comprendre sur le terrain comment ce facteur négatif agit (ou pas) dans les différents milieux et au sein des générations. L'affaiblissement de l'endogamie raciale (si tant est que ce concept ait un sens) doit ainsi être sérieusement étudiée. De même, la métaphore implicite d'une sorte de négociation compensatrice de la couleur contre la position sociale est sans doute un raccourci un peu rapide de la réalité des choses. Il conviendrait sans doute de chercher à expliquer pour chaque localité les évolutions statistiques en fonction des comportements réels des individus observés sur le terrain. Un corollaire à cette idée consiste à proposer de ne pas envisager les mariages mixtes sous l'angle de contacts entre " groupes raciaux ». La perspective utilisée pour les groupes ethniques, par exemple les Japonais dans les années $1960^{49}$, n'est pas appropriée pour envisager les unions entre noirs, métis et blancs. Les résultats de mon enquête de terrain étudiée plus haut montrent l'intérêt de regarder le degré de similitude physique chez les conjoints. D'autres enquêtes pourraient mesurer finement quartier par quartier pour plusieurs villes la « distance physique » et intégrer cette notion dans un indicateur plus fin de distance physique et sociale.

Enfin, il me semble évident que les situations de rencontre et de « namoro » comme disent les Brésiliens doivent être étudiées par les anthropologues. De telles études permettraient de mieux comprendre comment les caractéristiques et les qualités des individus reçoivent du sens en fonction du contexte ${ }^{50}$. Le point décisif en matière de mariage est bien la question de la décision menant à l'union. La façon dont la famille juge l'engagement des jeunes gens cherchant à se marier doit pouvoir révéler la variabilité des interprétations d'une génération à l'autre. Cette manière de procéder n'a rien de commun avec l'utilisation de questionnaire

\footnotetext{
${ }^{49}$ Les études sur les descendants de Japonais au Brésil avaient par exemple montré la plus stricte endogamie ethnique : la seule concession des familles étant d'abandonner quelques coutumes traditionnelles organisant les unions. Voir à ce sujet : Lucia Wollet de Mello, « Costumes matrimoniais entre Japones e seus descendentes », in : Egon Schaden (org.) Homem, cultura e sociedade no Brasil, Petrópolis, Vozes, 1972.

${ }^{50}$ J'emprunte la formulation à L. A. Rebhum, « Sexuality, color and stigma amonf Northeast brazilian women », Medical Anthropology Quarterly, 18, 2, 2006, pp. 183-199.
} 
d'attitudes demandant aux répondants s'ils «se marieraient avec un homme ou une femme plus foncés qu'eux », qui ne mesure que des situations imaginaires.

L'idée de fond qui soutient cet article est qu'il est particulièrement dangereux de confondre inférences globales à partir de données de recensement et dynamiques réelles des individus impliqués dans les unions sur le terrain. Les conséquences possibles de la méthodogenèse en matière d'études des relations raciales sont négligées alors qu'elles sont probablement considérables. L'étude des relations raciales au Brésil dispose d'un champ de progrès immense si elle accepte d'expliquer les recensements par des observations de terrain et non l'inverse. C'est dans le travail conjoint des qualitativistes et des quantitativistes, fondé sur le désir de faire des découvertes et pas sur celui d'appliquer un schéma militant préexistant que peuvent se trouver les meilleures voies de progrès de cette sociologie encore si mal fixée méthodologiquement au Brésil. 